American Journal of Pharmacology and Toxicology 3 (2): 193-200, 2008

ISSN 1557-4962

(C) 2008 Science Publications

\title{
Proposal for the Development of Adverse Drug Reaction Prediction Model
}

\author{
${ }^{1}$ Muaed J. Alomar, ${ }^{2}$ Anas A. Hourani and ${ }^{2}$ Syed A. Sulaiman \\ ${ }^{1}$ Department of Clinical Pharmacy, Faculty of Pharmacy, University Sians Malaysia (USM), \\ Penang State, Malaysia \\ ${ }^{2}$ Department of Clinical pharmacy, Ajman University of Science and Technology Network, \\ Fujairah, UAE
}

\begin{abstract}
Information technology, which uses the computer for data processing and decision-making, has invaded all kinds of sciences. Health informatics is one of these important areas. The Objective of this study is to develop a mathematical model that calculates the percentage of ADR occurrence using the data available from primary literature for a specific medication. A mathematical model was developed to calculate the percentage of adverse drug reaction occurrence for a specific patient, using specific patient factors. The expected effect is that these different patient factors will produce different adverse drug reactions in different percentages. The adverse drug reaction prediction model is developed using Microsoft access, forms are built and ready for the data to be collected and utilized from primary literature. Pharmacists and other medical specialists should give information technology more concern in order to develop a comprehensive decision support systems able to predict medication errors before they actually happen.
\end{abstract}

Key words: Pharmacoinformatics, ADR, prediction medication, error model pharmacy

\section{INTRODUCTION}

Information technology which uses the computer for data processing and decision making has invaded all kinds of sciences. Many of the industrialist and research scientists along with the academicians are seriously thinking about the need of information technology in pharmaceutical sciences including clinical pharmacy and pharmaceutical care. Information technology has been applied indirectly in the drug discovery process and patient care for a long period of time.

Pharmacy Informatics involves the study, design and implementation of information and information systems in Pharmacy.

The creation of computerized prescriptions and laboratory databases has greatly enhanced the ability of institutions and organizations to screen for known adverse drug reactions.

Morimoto et al..$^{[1]}$ illustrated that patients with a history of angiotensin-converting enzyme inhibitorinduced cough were 29 times more likely to develop a cough than those without this history. These factors were used to develop a model stratifying patients into 4 risk groups. They suggested developing a system able to predict adverse drug reactions before they happen using the factors collected from patients histories.

Eric et al.,${ }^{[2]}$ in a study published in the annals of Internal Medicine concluded that the overall rates of dispensing errors and potential adverse drug reactions substantially decreased after implementing bar code technology. However, the technology should be configured to scan every dose during the dispensing process.

Computers do not process information but merely process data. Only a human being is able to interpret the data so that it becomes information. There is a parallel between computer-assisted data entry and observation with the human senses. Computer processing also has some parallels with the thought processes in the human brain. This concerns only that part of the processing that can be structured and generalized. In patient care, computers cannot and should not replace thought processes in the human brain but should amplify the brain's capabilities. A computer can extend the brain's memory, increase its data processing capabilities and improve the accuracy and consistency of our data processing. Similar to the way that the human senses are amplified by a microscope or

Corresponding Author: Muaed J. Al Omar, Department of Clinical Pharmacy, Faculty of Pharmacy, Ajman University of Science and Technology Network, Fujairah, UAE P.O. Box 7826 Fujairah, United Arab Emirates Tel: 00971507157641 Fax: 0097192223176 
a stethoscope, the human brain can be amplified by a computer.

Bates et al. ${ }^{[3]}$ found that Physician computer order entry decreased the rate of non-intercepted serious medication errors by more than half, although this decrease was larger for potential adverse drug reactions than for errors that actually resulted in adverse drug reactions.

Gandhi et al. $^{[4]}$ indicated that basic computerized prescribing systems may not be adequate to reduce errors. More advanced systems with dose and frequency checking are likely needed to prevent potentially harmful errors.

Dasta, Greer and Speedie ${ }^{[5]}$ suggested that pharmacists need to become more involved with applications of technology to pharmacy. Properly implemented, computers can provide more time for pharmacists to use their cognitive skills in the delivery of pharmaceutical care.

The objectictive of this study is to develop a mathematical model that calculates the percentage of ADR occurrence using the data available in the previously done literature for specific medication.

\section{MATERIALS AND METHODS}

The calculation will depend on the collection of patient related factors that might affect the development of the adverse drug reaction.

Patient's factors and the development of adverse drug reactions: The classification of patient factors is very important for proper calculation. The classification is as follows: category, factor classified and factors.

We have two types of data, the data obtained from literature and the data we have classified for the purpose of this study. For example, the classification for age was into ten year periods (1-9, 10-19, 20-29 etc). All data collected from literature needs to be classified into specific categories. Classification is essential for the calculation.

During data collection, all of the possible differences among patients are called categories and these categories include factors related to them. These categories are, Age, Gender, Race, Diseases, Creatinine Clearance, Allergy, Drinking alcohol, Fetus age, Heart rate, Job, Maternity, Medications, Obesity, Place of angioedema, Smoking, Time of taking the medication (dosing), Symptoms after, Treatment period, Weight and as many categories and factors as possible. The more factors available, the more precise the results will be.
For example, gender is a category, male and female are the factors belong to that specific category.

Data processing and calculation: Microsoft access is used for the development of the model. Specific forms are data entry collected from literature.

Definitions: The following are definitions for the terms used in the study:

- Study name, is the main title of the study and it is taken from each article exactly as it appears in the publication

- Study patients, is the total number of patients enrolled in the study in each article

- Drug is the drug used in the study

- $\mathrm{ADR}$ is the adverse drug reaction reported by the article

- Patient ADR number, is the total number of patients with specific factors having a specific adverse drug reaction in each article

- Category, example, Age, sex, race etc.

- Factor classified, is the sub classification of category, e.g. Sex is a category and it is classified into male and female ...etc. The factor classified is the one considered for calculation

- Factor patient number, is the number of patients in a specific factor classified

- Drug dose, is the dose of the drug given to the patient and it is in $\mathrm{mg}$

- Dose frequency, is the number of times the drug is being given

Steps of calculation: The first step in the calculation is the normalization. It is assumed that all literature is equal, in that if the same study is carried out anywhere in the world with the same criteria, we will get the same results. The difference is assumed to be only controlled by patient factors and environmental factors, i.e. if all factors are unified, the incidence in all literature should be the same.

Normalization is to change all numbers of each study into a comparable number by multiplying all the numbers with normalized factor. The normalized factor equals the number for normalization divided by patient's number.

The assumed number for normalization is 1000 and it is chosen because it is around the average of number of patients enrolled in the studies. Normalized number is used in order to make all study patients equal, then the comparison and calculation will be logical and acceptable. Figure 1 clarifies the steps of normalization. 


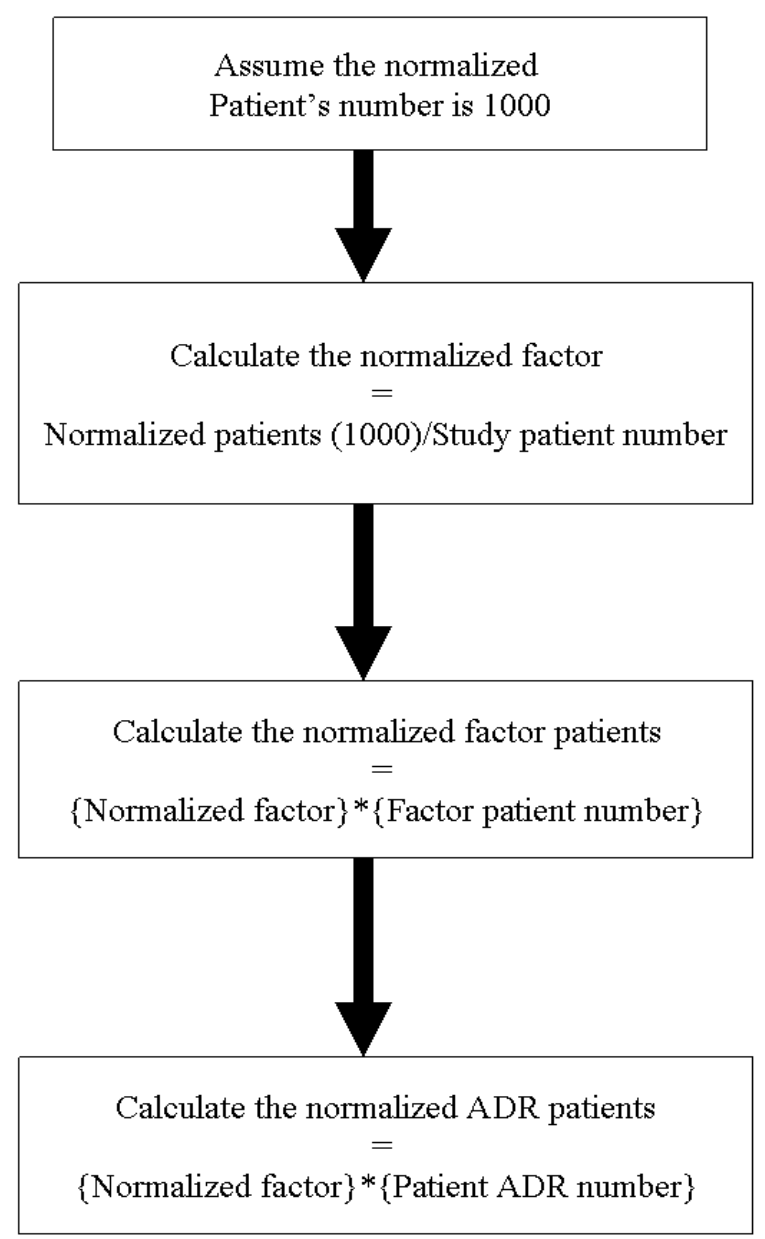

Fig. 1: Process of normalization

All of the numbers included in the study has to be changed in the same manner as the patient's numbers in order to be comparable. All of the numbers in the study must be multiplied with the factors used to normalize the number of patients.

\section{Steps of normalization:}

- Calculation of the normalized factor $=\{$ normalized patients 1000/study patient number\}. For the calculation we assume that all the studies have the same number of patients which is equal to 1000 and this is called normalized patients

- In order to compare between studies, the number of study patients should be equal, that's why normalization is done for the two important numbers, factor patient number and patient ADR number. Each number needs to be converted to be out of 1000 .
- Calculate the Normalized Factor Patients = [Normalized Factor]*[Factor Patient No]

- Calculate the Normalized ADR Patients = [Normalized Factor]*[Patient ADR No]

- This normalization must be done for every single factor in the study

Example 1: The following example will clarify normalization:

- Study name, Efficacy and Safety of Imidapril in Patients with Essential Hypertension

- Study Patients No $=29$

- Drug, Imidapril

- ADR, cough

- Patient ADR No, 4

- Category, age

- Factor Classified, 40-49 year

- Factor Patient No, 29

- Calculation for exampe1,

The number of normalized patients is 1000

- Normalized factor $=\{$ normalized patients $1000 /$ study patient number $\}$.

$=1000 / 29$

$=34.4827586206897$

- Calculate the Normalized Factor Patients = [Normalized Factor]*[Factor Patient No]

$=34.4827586206897 * 29$

$=1000$

The answer is not always 1000 but in this study the number of patients factor is equal to the total number of patients in the study.

- Calculate the Normalized ADR Patients = [Normalized Factor]*[Patient ADR No]

$=34.4827586206897 * 4$

$=137.931034482759$

The second step in the calculation is the calculation of the total normalized factor patients: Total normalized factor patients is the total of all patients sharing the same factor, the same adverse drug reaction and the same medication. It is the summation of all patients in all studies having the same criteria. The summation is accomplished after normalization. Factor classified is used for the calculation.

If factor patient number is the same as patient number, then the Total normalized factor patients for each study will 1000 and this is because of 
normalization. But if the number of patients included in the study are not the same as the ones in the factor, then the total normalized factor patients for each study will not be equal to 1000 .

Example 2: Fifty eight black patients have entered a study. 1 patient out of the 58 has developed second degree AV block.

- Drug: verapamil

- $\quad$ ADR, $2^{\text {nd }}$ degree AV block

- Patient ADR No, 1

- Category, Race

- Factor Classified, Black

- Factor Patient No, 58 (which means that all patients included in the study have the same factor

\section{Calculation:}

- $\quad$ Normalized patients $=1000$

- Normalized factor = normalized patients "1000"/study patient number\}. $=1000 / 58=17.2413793103448$

- $\quad$ Because factor patients $=58$

- Then, normalized factor patients $=$ [Normalized Factor]*[Factor Patient No] $=17.2413793103448 * 58=1000$

- Total normalized factor patients $=$ the summation of all normalized factor patients in all studies have the same factor (black patients)

- This calculation is done for only one factor at once

- Total normalized ADR Patients = [Total normalized Factor]*[Total Patient ADR No]

$17.2413793103448 * 1$

$=17.2413793103448$

- $\quad$ Percentage ADR patients = total normalized ADR patients/ total normalized factor patients*100\% $\{17.2413793103448 / 1000\} *\{100 \%\}$ 1.72413793103448

Example 3: In this example the study Patients Number doesn't equal factor:

- Patients No 3522 patients using Doxazosin GITS have entered a study.

- Drug: Doxazosin GITS

- ADR, Urinary incontinence

- Patient ADR No, 1

- Category, Gender

- Factor Classified, Female

- Factor Patient No, 1398

- Calculation:
- $\quad$ Normalized patients $=1000$

- Normalized factor $=$ normalized patients 1000 /study patient number $\}$.

- $\quad=1000 / 3522=0.283929585462805$

- Because factor patients $=1398$

- Then, normalized factor patients $=[$ Normalized Factor]*[Factor Patient No] $=0.283929585462805 * 1398=396.933560477002$

Total normalized factor patients $=$ the summation of all normalized factor patients in all studies have the same factor (black patients).

This calculation is done for only one factor at once.

- Total normalized ADR Patients = [Total normalized Factor]*[Total Patient ADR No] $0.283929585462805 * 1$ $=0.283929585462805$

- $\quad$ Percentage ADR patients = total normalized ADR patients/ total normalized factor patients $* 100 \%$ $\{0.283929585462805 / 396.933560477002\}$ $*\{100 \%\} 0.0715307582260372$

The third step in the calculation is the calculation of the total normalized ADR patients: Total Normalized ADR Patients is the total patients having the same ADR in the same row with the same specifications and it is calculated by summation of all studies having the same characteristics.

The fourth step in the calculation is the calculation of the percentage of normalized ADR patients: Percentage ADR patients = total normalized ADR patients/ total normalized factor patients $* 100 \%$. Total normalized ADR patients and total normalized factor patients must not be null (no missing data).i.e. missing data are not included in the calculation.

The first four steps are used for the calculation of percentage ADR patients for only one factor classified.

The fifth step in the calculation is the calculation of ADR percentage for more than one factor together: For the calculation of adverse drug reaction occurrence for a specific patient having different factors, e.g. age, sex, race...etc, these factors should be selected first and then the program will calculate the total percentage of adverse drug reactions occurrence for each factor separately. The resulted numbers after calculation will reflect the percentage of adverse drug reaction occurrence for all factors in separate way. The lowest percentage of them will be the minimum percentage of ADR occurrence and the highest one will be the maximum. 


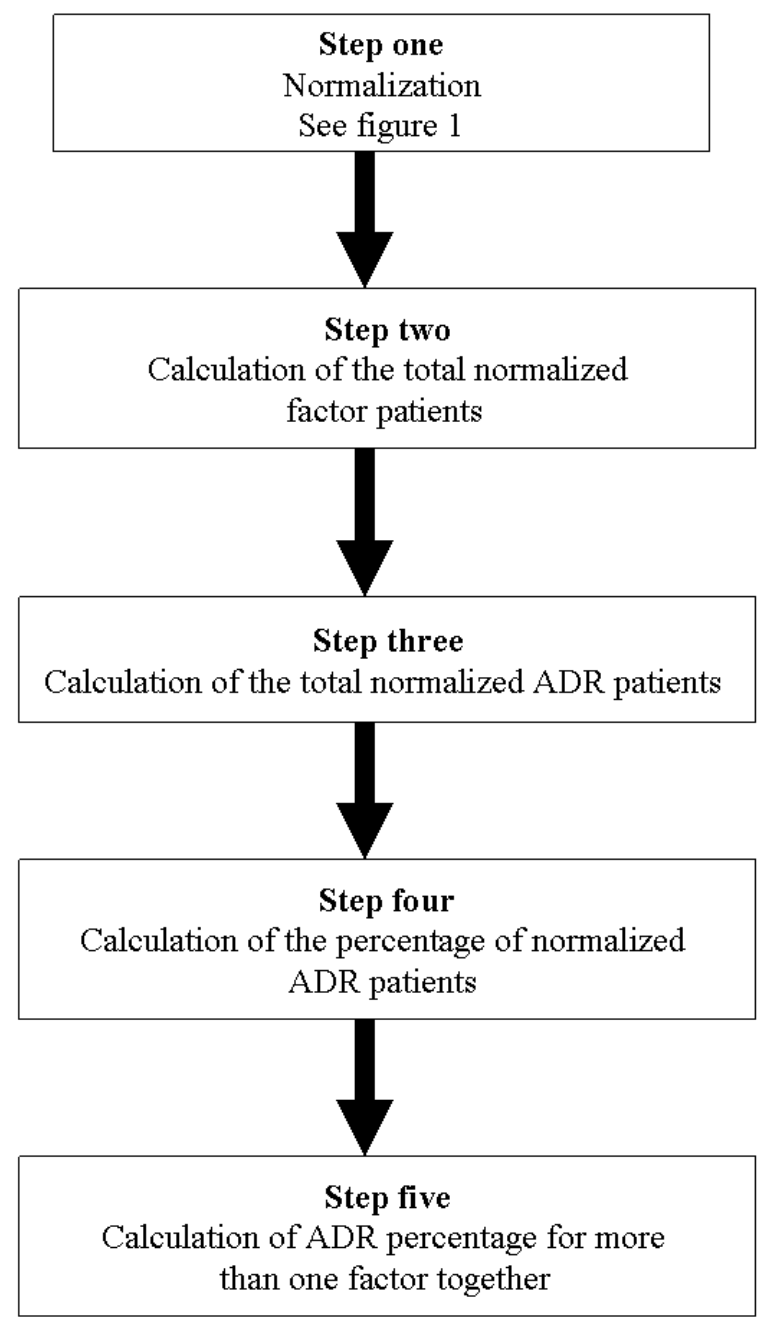

Fig. 2: Steps of calculation

If the minimum and the maximum percentage of ADR occurrence are equal, it most probably means that there is only one study available for the selected factors. Calculation steps are summarized in Fig. 2.

\section{RESULTS}

The end product of this research is the development of a new concept for the prediction of adverse drug reactions.

This program will calculate the minimum and the maximum percentage of adverse drug reaction occurrence in specific patient.

As a result of the new concept for the prediction of adverse drug reactions, different factors give different percentages for the minimum and the maximum occurrence of ADRs.
Model's Data entry form: Microsoft access forms are used for data entry. An example of data entry forms is shown in fig. 3 and fig.4. Data entry includes, the name of the study, the publication date, the number of the patients in the study, the location of the study in the program, drug name, drug dose, frequency of the dose, dosage form if available, brand name if available, the number of patients in each factor, the number of adverse drug reactions and the number of patients in each factors who developed the adverse drug reaction.

\section{DISCUSSION}

The implementation of information technology in health sciences is advocated to give hope for bringing a new strategy for better services and patient care.

A system is a collection of components that work together to achieve a common objective. The objective in the case of health information technology is to improve health care systems through optimal information support, i.e. it is a meaningful collection of facts or data that help in the proper treatment.

The World Health Organization (WHO) has long identified health information systems as important and crucial for achieving health for everybody. A report of a WHO meeting (1987) clearly links improved management to improved health information systems.

The creation of a huge amount of research is not the final goal in itself, but it is a means to provide better decisions in the medical practice, better designs for pharmacotherapeutic plans, better implementation and better follow up plans. After implementation, this information needs to be reevaluated and used in the best way possible.

Medical information is good data, which once available, will be transformed and converted into useful information which, in turn, will influence medical decisions, this kind of information will lead to decisions that are more effective and appropriate. This in turn leads to better practice and procedures and better patient care plans.

Information can only influence medical decisions if it is relevant, reliable and available for the decision maker in a timely fashion.

Some information can be used directly to produce decisions. For example the stock level of specific drug item can easily trigger a decision about the urgency of ordering the drug. However, in most instances, the direct use of raw data in this fashion is not feasible. Therefore, selecting appropriate indicators (factors) allows healthcare system professionals to transform crude information into a form that is more suited for decision making. Investing in developing health 
Am. J. Pharm. \& Toxicol., 3 (2): 193-200, 2008

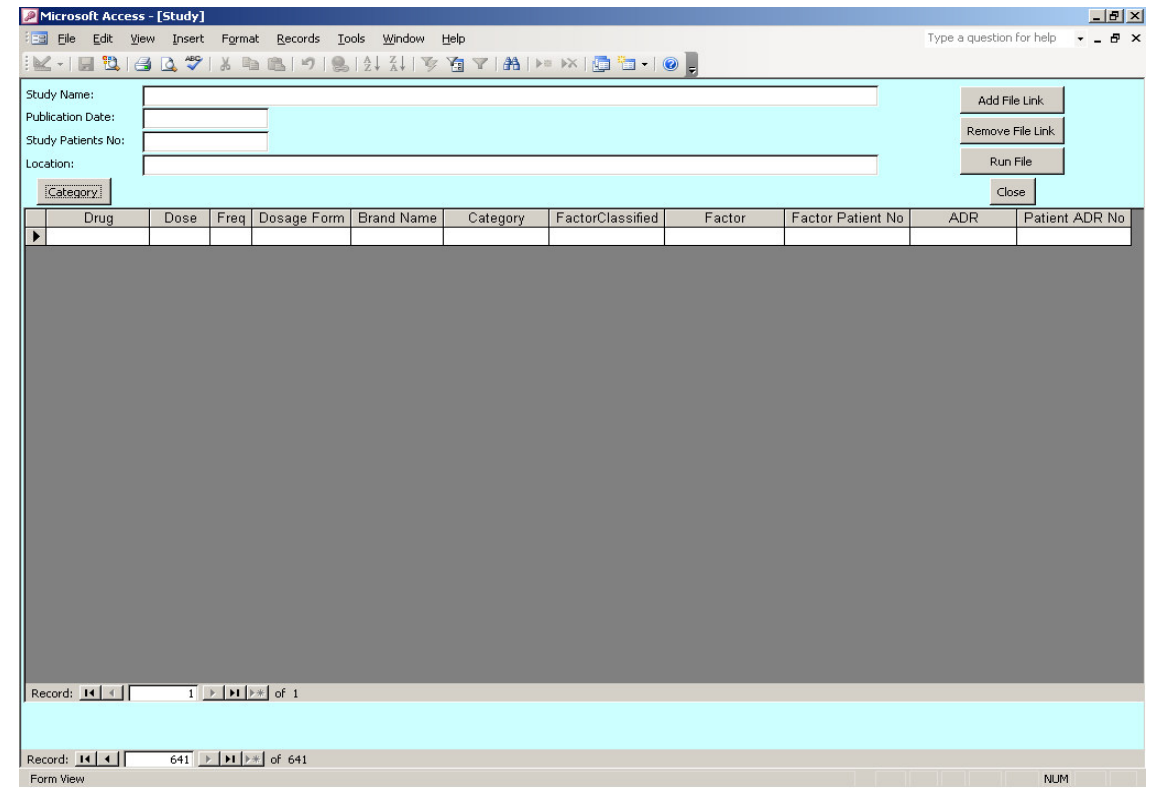

Fig. 3: Empty data entry form

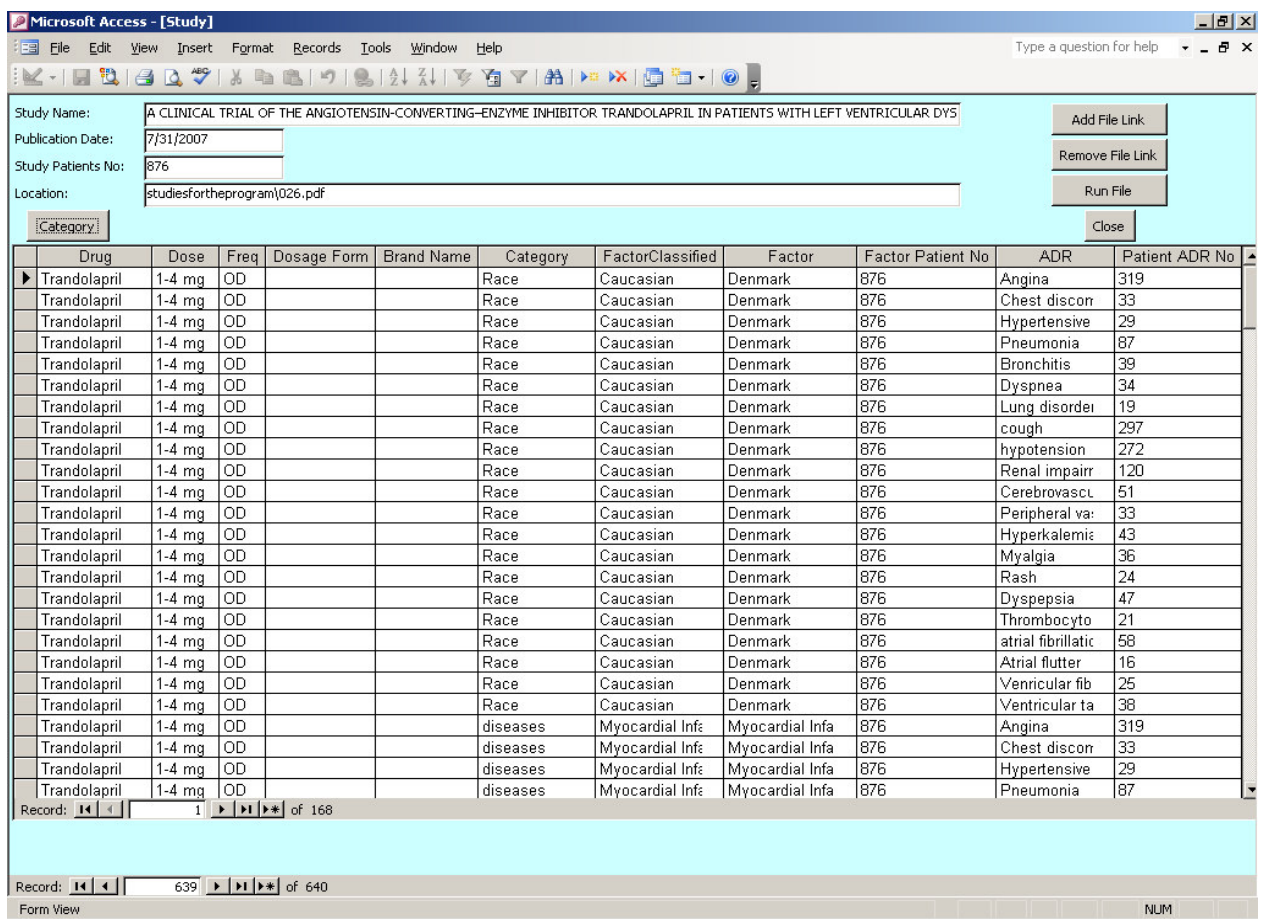

Fig. 4: Example of filled data entry form

information systems should be rewarding because it leads to an improved healthcare system and to build up and routine system that uses and produces information continuously, these information can be utilized for further development and research and even for helping in making appropriate medical decisions.

The adverse drug reaction prediction model is an example of what information technology can offer to 
the human kind by facilitating the analyses and helping in the utilization of the medical information.

Information technology depends on the accuracy of data collection and the availability of every possible factor that might affect decision making.

The more specific the information collected about patients, the more accurate the prediction will be. This adverse drug reaction prediction model depends solely on the data collected about the patients, everything including lifestyle, past medical history, past medication history, social history, type of work, smoking status, age, race, sex and any other factor which might influence human life. The concept of using factors in the detection of adverse drug reactions is because not every patient taking a certain medication will develop an ADR, this means that some people are vulnerable to ADRs more than others and this vulnerability depends on certain factors some people have and others do not. If these factors are used, then the exact differences between people will be more clear and we will be able to answer the question "Why do some people develop ADRs more than others?"

Incorporating these factors in information technology to develop a computer program which can calculate the expected ADR occurrence belongs to a science called Clinical pharmacoinformatics.

This program will open the door for more innovative clinical pharmacoinformative research thinking which enables us to utilize the information technology up to the maximum level for the sake of human safety.

This program is built on data collected from literature. This procedure has many drawbacks because not all of the information needed is available. The implementation of this program needs full cooperation from all the medical team in the hospitals and clinics and pharmacies on one side and the patient from the other side. Full cooperation ensures proper data collection and the accuracy in utilizing this data in the future. This program is part of a complete clinical pharmacoinformatic system which controls all the steps in the diagnosis and treatment of patients.

The medical team, including physicians, pharmacists, nurses and laboratory analysts should lean how to utilize the information technology available nowadays for their own benefit and to help them analyze and utilize the huge amount of the medical information available. No one person can handle all of this information, as it is beyond human capability.

It has to be acknowledged that healthcare providers might have some difficulty in accepting new technology to help them in their medical decision making process. Such rejection or barriers must be taken into consideration and must be handled carefully. This is because if the technology is implemented with unprofessional personnel, it might lead to more problems and medication errors. Full training for the medical teams are needed to enable them to utilize computer information technology in the best way possible $^{[6]}$.

Kristiina H., Kaija Sarantoa, \& Pirkko Nykanen, (2007) documented that the use of information systems was conductive to more complete and accurate documentation by healthcare professionals. This leads to the accumulation of high quality patient information services in the development of the healthcare process ${ }^{[7]}$.

Choosing the best drug for the right patient offers good health outcomes, saves money for the patient, saves money for the health institution and third parties, improves quality of life and encourages better healthcare practices for the other healthcare providers who have some skepticism towards the efficiency of technology in helping the decision makers in making their decisions. This issue is discussed by Bengt Sjo"borg et al. ${ }^{[8]}$.

\section{CONCLUSION}

Information technology is involved in everything in human life. The utilization of this kind of technology provides a promising future for the safe use of medications and helps in medical decision making and proper drug selection.

The objective of the study, is to develop a new Clinical pharmacoinformatics approach for the prediction of adverse drug reactions using the past medical data published in primary literature. In this manner it is possible to reach the optimal method of data collection by the cooperation between the patients and the medical institutions which makes the records more accurate and frequently updated.

This was achieved by creating the adverse drug reaction prediction model using mathematical models for the calculation of the possible minimum and maximum ADRs occurrence percentage.

The adverse drug reaction prediction model depends solely on the factors related to the patients including age, sex, race, past medical history, past medication history, lifestyle and all possible environmental factors which might affect human life.

It is recommended that all literature should be written in a clear way in which all of the needed data should be available to facilitate further research and make the medical decision more accurate and precise.

It is also recommended that pharmacists and physicians pay more attention to the technology 
available today and to use it frequently in order to obtain proper medical information with the highest possible level of care and accuracy.

These factors are collected and added carefully in a mathematical manner in order to produce a scientifically accepted number.

Medical decision depends partially or totally on literature. For that reason primary literature needs further evaluation. Perhaps the conditions could have been better, or better organization was required. By looking at the available literature we can analyze how different each one of them is, in the way of writing, the availability of basic data, in the results and the accuracy in showing those results.

\section{REFERENCES}

1. Morimoto, T., T.K. Gandhi, J.M. Fiskio, A.C. Seger, J.W. So, E.F. Cook, et al., 2004. Development and validation of a clinical prediction rule for angiotensin-converting enzyme inhibitorinduced cough. J. Gen. Inter. Med., 19: 684-691. Doi: 10.1111/j.1525-1497.2004.30016.x.

2. Churchill William, L. Cina Jennifer, Featherstone Erica, G. Patel Nirali, Poon Eric, and M. Rothschild Jeffrey. 2006. Medication dispensing errors and potential adverse drug events before and after implementing bar code technology in the pharmacy. Ann. Inter. Med., 145: 426-434. http://www.annals.org/cgi/content/abstract/145/6/42 6.

3. Bates, D.W., D.J. Cullen, N. Laird, L.L. Leape, L.A. Petersen, J.M. Teich, et al., 1998. Effect of computerized physician order entry and a team intervention on prevention of serious medication errors. JAMA., 280: 1311-1316. Doi: 10.1001/jama.280.15.1311.
4. Gandhi, T.K., J. Borus, E. Burdick, E.G. Poon, A.C. Seger and S.N. Weingart, 2005. Outpatient prescribing errors and the impact of computerized prescribing. J. Gen. Inter. Med. 20: 837-841. DOI: 10.1111/j.1525-1497.2005.0194.x.

5. Dasta, J.F. and M.L. Greer, 1992. Speedie SM. Computers in healthcare: overview and bibliography. Ann. Pharmacother, 26: 109-117. www.theannals.com/cgi/content/abstract/26/1/109.

6. Chisolm Deena, J., Kelleher Kelly, McAlearney Ann Scheck, A. Medow Mitchell and Schweikhart Sharon 2007. The story behind the story: Physician skepticism about. Int. J. Med. Inform., 76: 836842. doi:10.1016/j.ijmedinf.2006.09.021.

7. Kristiina, H., Kaija Sarantoa and Pirkko Nykanen, 2008. Definition, structure, content, use and impacts of electronic health records: A review of the research literature. Int. J. Med. Inform., 77: 291-304. http://www.ncbi.nlm.nih.gov/ pubmed/17951106.

8. Arvidsson Lars-Bertil, Backstrom Tobias, Bengt, Eiermann Birgit, Borg Bengt Sjo and Blomberg L. Karlsson Eva Andersen et al., 2007. Design and implementation of a point-of-care computerized system for drug therapy in Stockholm metropolitan health region-Bridging the gap between knowledge and practice. Int. J. Med. Inform., 76: 497-506. doi:10.1016/j.ijmedinf.2006.02.010. 